NORDITA-2002-60 HE, HIP 2002-39/TH

\title{
The inverted XY universality of the superconductivity phase transition
}

\author{
T. Neuhaus ${ }^{\mathrm{a}}$, A. Rajantie ${ }^{\mathrm{b}}$ and K. Rummukainen ${ }^{\mathrm{c}}$

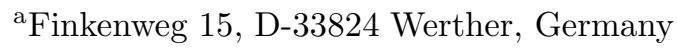

 \\ ${ }^{\mathrm{c} N O R D I T A, ~ B l e g d a m s v e j ~ 17, ~ D K-2100 ~ C o p e n h a g e n ~} \varnothing$, Denmark and Department of Physics, P.O.Box \\ 64, 00014 University of Helsinki, Finland
}

\begin{abstract}
It has been conjectured that the phase transition in the Ginzburg-Landau theory is dual to the XY model transition. We study numerically a particular limit of the GL theory where this duality becomes exact, clarifying some of the problems encountered in standard GL theory simulations. This may also explain the failure of the superconductor experiments to observe the XY model scaling.
\end{abstract}

\section{INTRODUCTION}

The (3-d) U(1) gauge + Higgs (GinzburgLandau, GL) theory is an effective theory for the superconductor-insulator phase transition. Despite the formal simplicity of the GL theory, and numerous analytical and numerical studies, the universal properties of the transition have not yet been fully resolved. Theoretical duality arguments [1] suggest that the phase transition is in the 3d XY model universality class, but with an inverted temperature axis. Thus, the symmetric and broken phases in the XY model correspond to the insulator and superconducting phases of the superconductor.

The duality gives concrete predictions for the behaviour of several critical observables in superconductors. For example, the Abrikosov vortex tension $\mathcal{T}$ should scale with the XY model exponent $\nu_{\mathrm{XY}}=0.6723$. Experimentally, it is easier to access the penetration depth $\lambda$ (or the inverse photon mass), which is also argued to scale with the $\mathrm{XY}$ exponent $\nu^{\prime}=\nu_{\mathrm{XY}}$.

However, the XY universality has not been unambiguously observed. Two different high- $T_{c}$ $\mathrm{YBa}_{2} \mathrm{Cu}_{3} \mathrm{O}_{7-\delta}$ experiments [3] report $\nu^{\prime}$ in the range $\approx 0.3 \ldots 0.5$. Monte Carlo simulations of the GL model favour $\nu^{\prime} \sim 0.3$ 国.2

\footnotetext{
$\overline{{ }^{1} \text { Accurate value of } \nu_{\mathrm{XY}}}$ can be found in Ref. [2

2 In the London limit (fixed length Higgs), where the du-
}

One reason for the confusing results both in experiments and numerical simulations is that the duality is expected to be valid only in a very narrow temperature range around the critical temperature, requiring extreme precision and large volumes in the measurements. Moreover, the duality relates "simple" observables (like the field expectation value in the XY model) to non-local observables in the dual theory (vortex network in the GL theory). This makes it very difficult to know whether the problems are caused by insufficient resolution near the critical temperature, or by the difficult nature of the observables themselves.

In order to gain insight into this problem we study a special limit of the GL theory, the frozen superconductor (FZS) (an integer-valued gauge theory), which is exactly dual to the XY-Villain model at all temperatures. Thus, the transition in FZS is bound to be in the XY model universality class. Studying the critical quantities of FZS can shed light on the problems faced in both the GL theory simulations and superconductor experiments. Detailed results are published in [6].

Our starting point is the lattice GL model in the London limit:

$\mathcal{L}_{\mathrm{GL}}=\frac{1}{2} \sum_{i<j} F_{\vec{x}, i j}^{2}+\kappa \sum_{i} s\left(\theta_{\vec{x}+i}-\theta_{\vec{x}}-q A_{\vec{x}, i}\right)$.

ality is on a firmer footing, simulations appear to give $\nu^{\prime} \approx 0.67$ 所. 

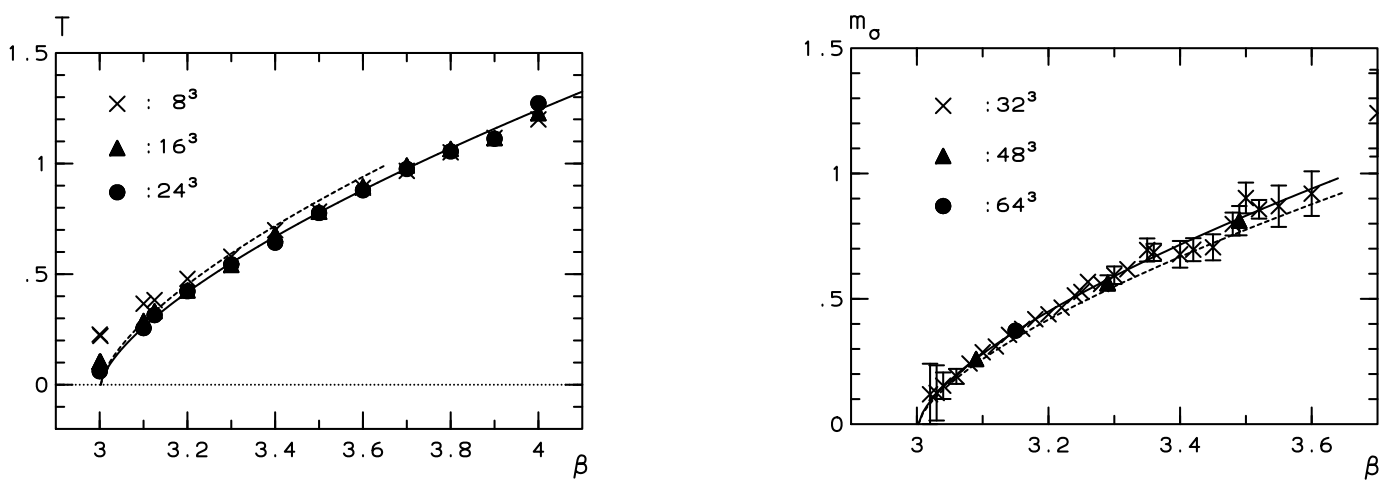

Figure 1. Vortex tension in FZS (left) and the scalar mass in the XY model (right), plotted against $\beta=1 / \kappa_{\mathrm{XY}}$. The continuous lines show power law fits, and, for comparison, the dashed lines show the fits transferred from the other plot.

Here $A_{x, i}$ is a real-valued gauge field, $\theta_{x}$ is the spin angle variable, and $F_{\vec{x}, i j}$ is the non-compact plaquette. We use the Villain form hopping term

$s(x)=-\ln \sum_{k=-\infty}^{\infty} \exp \left[-\frac{1}{2}(x-2 \pi k)^{2}\right]$.

We shall study the following 2 limiting cases of the GL model:

i) Let $\kappa \rightarrow \infty$ and define $\beta=4 \pi^{2} / q^{2}$. Now the GL model becomes the Frozen Superconductor (FZS):

$Z_{\mathrm{FZS}}(\beta)=\sum_{\left\{I_{\vec{x}, i}\right\}} \exp \left(-\frac{\beta}{2} \sum_{\vec{x}, i>j} \square_{\vec{x}, i j}^{2}\right)$.

Here $\square_{\vec{x}, i j}=I_{\vec{x}, i}+I_{\vec{x}+i, j}-I_{\vec{x}+j, i}-I_{\vec{x}, j}$, and the link variables $I_{\vec{x}, i}$ take integer values.

ii) Let $q \rightarrow 0$, and the GL model becomes the $\mathrm{XY}$ model with the Villain action

$Z_{\mathrm{XY}}(\kappa)=\int D \theta \exp \left(-\kappa \sum_{\vec{x}, i} s\left(\theta_{\vec{x}+i}-\theta_{\vec{x}}\right)\right)$.

The frozen superconductor and the XY-Villain model are exactly dual to each other with the identification $\beta=1 / \kappa$, i.e.

$Z_{\mathrm{XY}}(\kappa)=Z_{\mathrm{FZS}}(\beta=1 / \kappa)$.

This relation is valid at infinite volume; on a finite volume there are corrections proportional to the surface area of the volume. For a proof of this relation see [6].

\section{CRITICAL OBSERVABLES}

The XY-Villain model has a symmetry breaking transition at $\kappa=\kappa_{c} \approx 0.333068(7)$ [6]. Because of the exact duality, FZS must have a transition at $\beta_{c}=1 / \kappa_{c}$, which is of XY model universality, and the phases are related as follows:

\begin{tabular}{ll}
\hline XY model: & $\leftrightarrow$ FZS: \\
symmetric $\kappa<\kappa_{c}$ & $\leftrightarrow$ superconducting $\beta>\beta_{c}$ \\
broken $\kappa>\kappa_{c}$ & $\leftrightarrow$ Coulomb $\beta<\beta_{c}$ \\
\hline
\end{tabular}

\section{Vortex tension}

The duality implies that the XY model scalar correlation function equals the FZS AbrikosovNielsen-Olesen "vortex operator." In particular, the XY model scalar mass $m$ equals the tension $\mathcal{T}$ of the vortex line between a monopoleantimonopole pair in FZS. Thus, in the symmetric/superconducting phase of the XY/FZS model we should find

$m(\kappa)=\mathcal{T}(1 / \kappa) \propto\left|\kappa-\kappa_{c}\right|^{\nu_{\mathrm{XY}}}$,

and in the broken/Coulomb phases $m=\mathcal{T}=0$.

This is indeed the case: in Fig. 2 we show the results for $\mathcal{T}$ and $m$. As predicted by duality, $\mathcal{T}=m$ within the statistical errors, and the tension critical exponent is $0.672(9)$, compatible with $\nu_{\mathrm{XY}}$.

\section{Photon mass}

The most natural observable for the FZS model (and the one usually measured in high- $T_{c}$ superconductor experiments) is the photon mass 


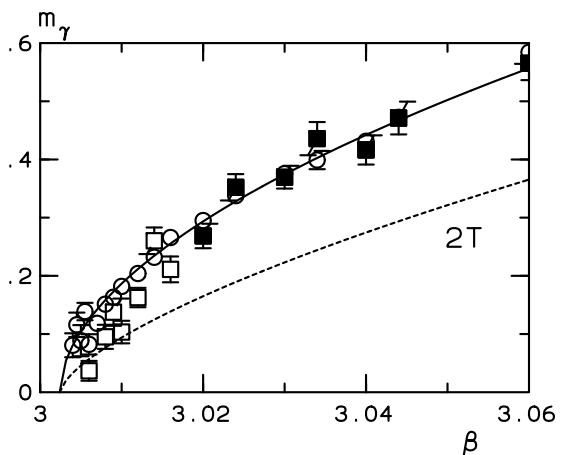

Figure 2. Photon mass measured from the FZS model in close proximity to the critical point. The continuous line is a power law fit, and the dashed line shows the curve $2 \times \mathcal{T}$.

$m_{\gamma}=1 / \lambda$, the inverse of the penetration depth. The duality relates $\lambda$ to the correlation length of the Noether current operator in the XY-Villain model. Parametrizing the critical behaviour of $m_{\gamma}$ with the exponent $\nu^{\prime}$, we have $m_{\gamma} \propto\left|\beta-\beta_{c}\right|^{\nu^{\prime}}$ in the superconducting phase. The theoretical prediction is $\nu^{\prime}=\nu_{\mathrm{XY}}[7]$.

Fig. 2 shows the $m_{\gamma}$ values measured from the plaquette correlation functions in FZS. A power law fit yields $\nu^{\prime} \approx 0.54$, not compatible with the prediction from the duality. However, this value agrees with (one of) the experimental results [3] and Monte Carlo simulations of the GL theory 6]. The dashed line shows $2 \times \mathcal{T}$. Since the photon operator we use couples to two vortices, and the measured $m_{\gamma}>2 \times \mathcal{T}$, we conclude that the observed $m_{\gamma}$ shows pre-asymptotic behaviour, and the true asymptotic value is $m_{\gamma}=2 \times \mathcal{T} \propto$ $\left(\beta-\beta_{c}\right)^{\nu_{\mathrm{XY}}}$, which agrees with the duality.

\section{Anomalous dimension}

At the critical point $\left(\beta=\beta_{c}\right)$, the photon (plaquette) correlation function is predicted to have a large anomalous dimension $\eta_{A}=1$ [7]

$\langle\square(-\vec{p}) \square(\vec{p})\rangle \propto|\vec{p}|^{\eta_{A}}$, when $|\vec{p}| \rightarrow 0$.

This is indeed what we observe: at $\beta_{c}$ the correlation function in FZS shows a clear power law dependence, with the power $\eta_{A}=0.98(4)$.

\section{CONCLUSIONS}

Because of the exact duality between the frozen superconductor (FZS) and the XY model, the critical observables in FZS must behave according to the corresponding XY model critical exponents. We have measured several observables in the frozen superconductor, and we duly find the behaviour predicted by the duality (for quantities other than reported here, see [6]). The sole exception is the photon mass $m_{\gamma}$, which appears to scale with an exponent substantially smaller than the XY model prediction. Exactly analogous behaviour has been observed superconductor experiments and in GL theory simulations.

Since the observed behaviour of $m_{\gamma}$ differs from predictions even when the duality is exact, the similar discrepancies observed in other studies do not mean that the duality hypothesis is not valid in real superconductors. The apparent incorrect scaling behaviour may be due to the large anomalous dimension $\eta_{A}$ of the photon propagator.

\section{REFERENCES}

1. M. E. Peskin, Annals Phys. 113 (1978) 122; C. Dasgupta and B.I. Halperin, Phys. Rev. Lett. 47 (1981) 1556; A. Kovner, P. Kurzepa and B. Rosenstein, Mod. Phys. Lett. A 8 (1993) 1343 [Erratum-ibid. A 8 (1993) 2527]; M. Kiometzis, H. Kleinert and A. M. J. Schakel, Phys. Rev. Lett. 73 (1994) 1975.

2. M. Campostrini et al., Phys. Rev. B63 (2001) 214503.

3. S. Kamal et al., Phys. Rev. B58 (1998) R8933; K. M. Paget et al., Phys. Rev. B59 (1999) 6545.

4. K. Kajantie, M. Laine, T. Neuhaus, A. Rajantie and K. Rummukainen, Nucl. Phys. Proc. Suppl. 106 (2002) 959.

5. P. Olsson and S. Teitel, Phys. Rev. Lett. 80 (1998) 1964; J. Hove and A. Sudbo, Phys. Rev. Lett. 84 (2000) 3426.

6. T. Neuhaus, A. Rajantie and K. Rummukainen, cond-mat/0205523.

7. I. F. Herbut and Z. Tesanovic, Phys. Rev. Lett. 76 (1996) 4588; B. Bergerhoff, F. Freire, D. Litim, S. Lola and C. Wetterich, Phys. 
Rev. B 53 (1996) 5734. 